\title{
Isolation, identification, and biological evaluation of Nrf2-ARE activator from the leaves of green perilla (Perilla frutescens var. crispa f. viridis)
}

\section{$\operatorname{AUTHOR}(\mathrm{S})$ :}

Izumi, Yasuhiko; Matsumura, Atsuko; Wakita, Seiko; Akagi, Ken-Ichi; Fukuda, Hiroyuki; Kume, Toshiaki; Irie, Kazuhiro; ... Sugimoto, Hachiro; Hashimoto, Tadashi; Akaike, Akinori

\section{CITATION:}

Izumi, Yasuhiko ... [et al]. Isolation, identification, and biological evaluation of Nrf2-ARE activator from the leaves of green perilla (Perilla frutescens var. crispa f. viridis). Free radical biology \& medicine 2012, 53(4): 669-679

\section{ISSUE DATE:}

2012-06-27

URL:

http://hdl.handle.net/2433/159047

\section{RIGHT:}

(c) 2012 Elsevier Inc.; この論文は出版社版でありません。引用の際には 出版社版をご確認ご利用ください。; This is not the published version. Please cite only the published version. 
Isolation, identification, and biological evaluation of Nrf2-ARE activator from the leaves of green perilla (Perilla frutescens var. crispa f. viridis)

Yasuhiko Izumi ${ }^{1}$, Atsuko Matsumura ${ }^{1}$, Seiko Wakita ${ }^{1}$, Ken-ichi Akagi $^{2}$, Hiroyuki Fukuda ${ }^{3}$, Toshiaki Kume ${ }^{1}$, Kazuhiro Irie ${ }^{4}$, Yuki Takada-Takatori ${ }^{5}$, Hachiro Sugimoto ${ }^{6}$, Tadashi Hashimoto ${ }^{3}$, and Akinori Akaike ${ }^{1,7}$

${ }^{1}$ Department of Pharmacology, Graduate School of Pharmaceutical Sciences, Kyoto University, 46-29 Shimoadachi-cho, Sakyo-ku, Kyoto 606-8501, Japan

${ }^{2}$ Section of Laboratory Equipment, National Institute of Biomedical Innovation, 7-6-8 Asagi Saito, Ibaraki 567-0085, Japan

${ }^{3}$ Theravalues Corporation, 1st floor, Kioicho Building, 3-12 Kioicho, Chiyoda-ku, Tokyo 102-0094, Japan

${ }^{4}$ Laboratory of Organic Chemistry in Life Science, Division of Food Science and Biotechnology, Graduate School of Agriculture, Kyoto University, Kitashirakawa-Oiwake-cho, Sakyo-ku, Kyoto 606-8502, Japan

${ }^{5}$ Department of Pharmacology, Faculty of Pharmaceutical Sciences, Doshisha Women's

College, Kodo, Kyotanabe, Kyoto 610-0395, Japan

${ }^{6}$ Graduate School of Life and Medical Sciences, Doshisha University, 1-3 Tataramiyakodani, Kyotanabe, Kyoto 610-0321, Japan

${ }^{7}$ Graduate School of Pharmaceutical Sciences, Nagoya University, Furo-cho, Chikusa-ku, Nagoya 464-8601, Japan 
Running title: Identification of Nrf2-ARE activator from green perilla.

Address correspondence to:

Akinori Akaike, Ph.D.

Department of Pharmacology, Graduate School of Pharmaceutical Sciences,

Kyoto University

46-29 Yoshida-shimoadachi-cho, Sakyo-ku, Kyoto 606-8501, Japan

Phone: +81-75-753-4550 FAX: +81-75-753-4579

E-mail: aakaike@pharm.kyoto-u.ac.jp

Abbreviations: antioxidant response element (ARE), 2',7’-dichlorofluorescein (DCF), 2',3'-dihydroxy-4',6'-dimethoxychalcone (DDC), dihydroethidium (DHE), dimethylsulfoxide (DMSO), electron impact (EI), ethidium (ETH), $\gamma$-glutamylcysteine synthetase ( $\gamma$-GCS), reduced glutathione (GSH), 2',7'-dichlorodihydrofluorescein diacetate ( $\left.\mathrm{H}_{2} \mathrm{DCFDA}\right)$, heme oxygenase-1 (HO-1), high-pressure liquid chromatography (HPLC), 6-hydroxydopamine (6-OHDA), kelch-like ECH-associated protein 1 (Keap1), mitogen-activated protein kinase (MAPK),mass spectrometry (MS), 3-(4,5-dimethylthiazol-2-yl)-2,5-diphenyltetrazolium bromide (MTT), nuclear magnetic resonance (NMR), NAD(P)H: quinone oxidoreductase-1 (NQO1), nuclear factor erythroid 2-related factor 2 (Nrf2), oxygen radical absorbance capacity (ORAC), phosphoinositide 3-kinase (PI3K), reactive oxygen species (ROS), small interfering RNA (siRNA), trifluoroacetic acid (TFA). 


\section{Abstract}

The nuclear factor erythroid 2-related factor 2 (Nrf2)-antioxidant response element (ARE) pathway is a cellular defense system against oxidative stress. Activation of this pathway increases expression of antioxidant enzymes. Epidemiological studies have demonstrated that the consumption of fruits and vegetables is associated with reduced risk of contracting a variety of human diseases. The aim of this study is to find Nrf2-ARE activators in dietary fruits and vegetables. We first attempted to compare the potency of ARE activation in six fruit and six vegetables extracts. Green perilla (Perilla frutescens var. crispa f. viridis) extract exhibited high ARE activity. We isolated the active fraction from green perilla extract through bioactivity-guided fractionation. Based on nuclear magnetic resonance and mass spectrometric analysis, the active ingredient responsible for the ARE activity was identified as 2',3'-dihydroxy-4',6'-dimethoxychalcone (DDC). DDC induced the expression of antioxidant enzymes, such as $\gamma$-glutamylcysteine synthetase ( $\gamma$-GCS), NAD(P)H: quinone oxidoreductase-1 (NQO1), and heme oxygenase-1. DDC inhibited the formation of intracellular reactive oxygen species and the cytotoxicity induced by 6-hydroxydopamine. Inhibition of the p38 mitogen-activated protein kinase pathway abolished ARE activation, the induction of $\gamma$-GCS and NQO1, and the cytoprotective effect brought about by DDC. Thus, this study demonstrated that DDC contained in green perilla enhanced cellular resistance to oxidative damage through activation of Nrf2-ARE pathway.

Keywords: chalcone; green perilla; Nrf2-ARE pathway; oxidative stress; 6-hydroxydopamine. 


\section{Introduction}

Oxidative stress, also referred to as a reactive oxygen species (ROS)-antioxidant imbalance, occurs when the net amount of ROS exceeds the antioxidant capacity. Oxidative stress is thought to play a major role in the pathogenesis of a variety of human diseases, including aging, carcinogenesis, metabolic syndrome, cardiovascular, neurodegenerative and kidney diseases [1], [2], [3], [4], [5], and [6]. Epidemiological evidence indicates that a significant reduction in the risk of ischemic stroke, some forms of cancer, and Alzheimer's disease can be obtained by increasing fruit and vegetable consumption [7], [8] and [9]. Therefore, it is widely believed that dietary fruit and vegetable intake is beneficial in preventing disease onset and slowing disease progression.

Vitamins C and E, polyphenols, and carotenoids are thought to be responsible for most of the antioxidant activity in foods. Various studies have suggested that dietary antioxidants may protect against cardiovascular diseases, neurodegenerative diseases, and some forms of cancer [10], [11], [12], and [13]. The direct antioxidant capacities of food extracts have been comparatively assessed using TEAC (Trolox Equivalent Antioxidant Capacity), FRAP (Ferric Reducing Ability of Plasma), and ORAC (Oxygen Radical Absorbance Capacity) assays [14], [15], and [16]. Although the radical-scavenging activities of phytochemicals from foods are responsible, at least in part, for human health-promoting effects, it is unclear whether the direct antioxidant activities of phytochemicals in vitro can completely explain their systemic antioxidant effects in vivo [17].

Accumulating evidence suggests that many phytochemicals, such as sulforaphane and curcumin, enhance the expression of antioxidant enzymes and cytoprotective proteins, for 
example, NAD(P)H: quinone oxidoreductase-1 (NQO1), superoxide dismutase, glutathione S-transferase, glutathione peroxidase, heme oxygenase-1 (HO-1), $\gamma$-glutamylcysteine synthetase ( $\gamma$-GCS), catalase, and thioredoxin [18]. The enhanced expression of antioxidant enzymes and cytoprotective proteins is mainly controlled by the nuclear factor erythroid 2-related factor 2 (Nrf2)-antioxidant response element (ARE) pathway. Under normal physiological conditions, Nrf2 is inactive due to binding with the skeletal actin-binding protein, Kelch-like ECH-associated protein 1 (Keap1). Under conditions of oxidative stress, Nrf2 is no longer sequestered by Keap1 and is subsequently translocated to the nucleus and bound to ARE. This results in the transcriptional activation of a number of phase 2 detoxifying and antioxidant enzymes [19]. Nrf2-ARE pathway functions as a cellular defense system against oxidative stress and has recently received attention as a potential therapeutic target for cancer chemoprevention, and cardiovascular and neurodegenerative diseases [20], [21], and [22]. Nevertheless, compared to ORAC values in the database of the U.S. Department of Agriculture's Agricultural Research Service, limited data exists comparing this indirect antioxidant property among foods, although the potency of induction of NQO1 in selected vegetables has been examined [23].

To discover novel Nrf2-ARE activators, the present study was designed to compare the ARE activity of the extracts of fruits and vegetables that are widely consumed in either Eastern or Western food cultures. Fruits and vegetables tested included the following: peach (Amygdalus persica), apple (Malus pumila Mill.), strawberry (Fragaria L.), cranberry (Vaccinium oxycoccos), raspberry (Rubus idaeus), satsuma mandarin (Citrus unshiu Marc.), green perilla (Perilla frutescens var. crispa f. viridis), tossa jute (Corchorus olitorius L.), crown daisy (Glebionis coronaria L.), celery (Apium graveolens var. dulce), parsley (Petroselium crispum), 
and red perilla (Perilla frutescens var. crispa f.purpurea). We carried out a bioactivity-guided fractionation to isolate the active constituents from the fruit and vegetable extracts. After chemical identification, the active compounds were evaluated for their protective activity against 6-hydroxydopamine (6-OHDA)-induced cell toxicity. 


\section{Materials and Methods}

\section{Materials}

Fruit and vegetable samples were obtained from Ehime Beverage (Matsuyama, Japan), Skylight Biotech (Akita, Japan), and Sanyo Foods (Tokyo, Japan). 6-Hydroxydopamine hydrochloride and trans-cinnamaldehyde were purchased from Sigma (St. Louis, MO, USA). trans-Cinnamoyl chloride and (E)-chalcone were obtained from Wako (Osaka, Japan). 2'-Hydroxychalcone was obtained from TCI (Tokyo, Japan). 2',4'-Dimethoxychalcone, 2'-hydroxy-4',6'-dimethoxyacetophenone, 2'-hydroxy-4'-methoxychalcone, 2'-hydroxy-6'-methoxychalcone, and 2',6'-dimethoxychalcone were purchased from Indofine Chemical (Hillsborough, NJ, USA). SB203580 and LY294002 were obtained from Calbiochem (San Diego, CA, USA). Flavokawain B was purchased from LKT (St. Paul, MN, USA). 2',3'-Dihydroxy-4',6'-dimethoxychalcone was synthesized and provided by Pharmaeight (Kyoto, Japan).

\section{Cell culture, transfection, and luciferase reporter analysis}

Rat adrenal pheochromocytoma PC12 cells were maintained in Dulbecco's modified Eagle medium supplemented with 5\% fetal calf serum and 10\% horse serum. Cell cultures were incubated at $37^{\circ} \mathrm{C}$ in a humidified atmosphere of $95 \%$ air and $5 \% \mathrm{CO}_{2}$.

PC12 reporter cells were generated by stable transfection of ARE-luciferase construct. The annealed oligonucleotide of the rat NQO1 ARE (top strand:

CTCAGAGATTTCAGTCTAGAGTCACAGTGACTTGGCAAAATCA; bottom strand: CATGGAGTCTCTAAAGTCAGATCTCAGTGTCACTGAACCGTTTTAGTTCGA) was 
ligated to the KpnI and HindIII site of the pGL4.27 vector (Promega, Madison, WI, USA). The cells were transfected with the plasmid using Lipofectamine 2000 (Invitrogen, Carlsbad, CA, USA) according to the manufacturer's instructions. The culture medium was supplemented with hygromycin B (300 $\mu \mathrm{g} / \mathrm{ml})$ to select drug-resistant stable transfectants. In the reporter gene assays, firefly luciferase activity in cell lysates was measured with a luminometer using Picagene LT 2.0 Luminescence Reagent (Toyo Ink, Tokyo, Japan).

\section{Preparation of fruit and vegetable extracts}

The extraction solvent chosen in this study was diethylether, which is useful for extracting low molecular weight compounds [24]. Raw leaves (100 g) were oven dried at $75^{\circ} \mathrm{C}$ and pulverized, and then were suspended in distilled water (200 mL). Ether/water extraction was performed by adding an equal volume of diethylether to the aqueous mixture from juice (100 mL) or raw leaves (200 mL) and rigorously shaking for a few minutes using a separating funnel. The ether layer was evaporated to dryness in a vacuum rotary evaporator. Solvent extraction was repeated at least thrice. The residues were transferred to a glass tube and evaporated to dryness under nitrogen gas. The extracts were dissolved in dimethylsulfoxide (DMSO) and filtered through a 0.22- $\mu \mathrm{m}$ membrane.

The extraction solvent chosen in some cases was ethanol, which is generally recognized as safe. Raw leaves (100 g) were oven dried at $75^{\circ} \mathrm{C}$ and pulverized, and then immersed in ethanol solution (100 mL) for 2 days. The residues were re-extracted with ethanol solution (100 mL) for a further 3 days. The combined extract solutions were concentrated using a rotary evaporator under vacuum. The residues were evaporated to dryness under nitrogen gas 
and freeze-dried. The dried extracts were dissolved in ethanol and filtered through a 0.22- $\mu \mathrm{m}$ membrane.

\section{Purification of the active compound}

The ether extract was fractionated with a silica gel column chromatography $(60 \times 5$ mm i.d., $70-230$ mesh) and eluted with $n$-hexane-ethyl acetate $(25 \% \rightarrow 50 \% \rightarrow 75 \% \rightarrow 100 \%$ ethyl acetate, each $3 \mathrm{~mL}$ ). Twelve fractions were collected in $1 \mathrm{~mL}$ volumes. The fractions were evaporated to dryness under nitrogen gas. The residues were dissolved in DMSO.

Separation of the ether extract was also performed by high-pressure liquid chromatography (HPLC) with UV detection using a YMC-Pack Pro C18 column (150 × 4.6 mm i.d., with $5 \mu \mathrm{m}$ particle size) (YMC, Kyoto, Japan) using a flow-rate of $1 \mathrm{~mL} / \mathrm{min}$, at a temperature of $40^{\circ} \mathrm{C}$. Mobile-phase A consisted of 99\% (v/v) distilled water, $1 \%(\mathrm{v} / \mathrm{v})$ acetonitrile, and 0.1\% (v/v) trifluoroacetic acid (TFA). Mobile-phase B consisted of 99\% (v/v) acetonitrile, $1 \%(\mathrm{v} / \mathrm{v})$ distilled water, and $0.1 \%(\mathrm{v} / \mathrm{v})$ TFA. The injected volume was $50 \mu \mathrm{L}$ and the detection was performed at a wavelength of $300 \mathrm{~nm}$. The eluates were collected in volumes of $1 \mathrm{~mL}$ for $1 \mathrm{~min}$ each. The fractions were evaporated to dryness under nitrogen gas and freeze-dried. The residues were dissolved in DMSO.

\section{Nuclear magnetic resonance (NMR) and mass spectrometry (MS)}

The structure of the isolated compound was chemically identified by NMR and mass spectrometry. NMR spectra were recorded using an Avance II 800US² spectrometer (Bruker BioSpin, Rheinstetten, Germany) at $800 \mathrm{MHz}$ in DMSO- $d_{6}$. Chemical shifts for ${ }^{1} \mathrm{H}$ - and 
${ }^{13} \mathrm{C}-\mathrm{NMR}$ were referenced to tetramethylsilane (0.00 ppm). Assignments were made via

${ }^{1} \mathrm{H}-\mathrm{NMR},{ }^{13} \mathrm{C}-\mathrm{NMR}$, distortionless enhancement by polarization transfer (DEPT), total correlation spectroscopy (TOCSY), heteronuclear multiple quantum coherence (HMQC) and heteronuclear multiple-bond correlation (HMBC). High-resolution electron impact (EI)-MS analysis was performed on a JEOL JMS-600H mass spectrometer (Tokyo, Japan).

\section{Evaluation of cell viability}

The cell viability of PC12 cells was determined by 3-(4,5-dimethylthiazol-2-yl)-2,5-diphenyltetrazolium bromide (MTT) assays. The culture medium was replaced with serum-free medium containing $0.5 \mathrm{mg}$ / $\mathrm{mL}$ MTT tetrazolium salt (Nacalai Tesque, Kyoto, Japan) and incubated at $37^{\circ} \mathrm{C}$ for $30 \mathrm{~min}$. The medium was removed and the cells were scraped and solubilized into a 2-propanol solution. Aliquots were transferred to a 96-well plate and the absorbance was measured at $570 \mathrm{~nm}$. The viability of the cultures was expressed as a percentage of the absorbance measured in control cells.

\section{Small interfering RNA (siRNA) sequences and transfection}

siRNAs were purchased from Invitrogen. The siRNA sequence targeting Nrf2 \#1 was 5'-UUAAGACACUGUAACUCGGGAAUGG-3' and Nrf2 \#2 was 5'-UUUAAGUGGCCCAAGUCUUGCUCCA-3'. Stealth"TM RNAi Negative Control Medium GC Duplex \#2 (Invitrogen) was used as a negative control siRNA. PC12 cells in 35 mm culture dishes were transfected with each siRNA (200 pmol) using 7.5 $\mu$ L Lipofectamine 2000 according to the manufacturer's protocol. The medium was changed after $9 \mathrm{~h}$ and cultures were 
incubated for further $48 \mathrm{~h}$.

\section{Real-time PCR analysis}

Total RNA was extracted using a FastPure ${ }^{\circledR}$ RNA kit (Takara, Shiga, Japan). Total RNA was reverse-transcribed using a PrimeScript ${ }^{\circledR}$ RT-PCR kit (Takara). Real-time PCR was performed using SYBR ${ }^{\circledR}$ Premix Ex Taq ${ }^{\mathrm{TM}}$ II (Takara). The final solution contained $0.4 \mu \mathrm{M}$ primers and $2 \mu \mathrm{L}$ of cDNA in a total volume of $25 \mu \mathrm{L}$. The protocol involved denaturation at $95^{\circ} \mathrm{C}$ for $30 \mathrm{sec}$, and amplification $\left(40\right.$ cycles: $95^{\circ} \mathrm{C}$ for $5 \mathrm{sec}, 60^{\circ} \mathrm{C}$ for $30 \mathrm{sec}$ ). The primer sequences were Nrf2 forward 5'- GAGACGGCCATGACTGAT-3', Nrf2 reverse 5'-GTGAGGGGATCGATGAGTAA-3', glyceraldehyde 3-phosphate dehydrogenase (GAPDH) forward 5'-ATGGGAAGCTGGTCATCAAC-3', and GAPDH reverse 5'-GATCTCGCTCCTGGAAGATG-3'. The mRNA levels of tested genes were quantified using standard curves generated by serially diluted reference samples. The relative levels of Nrf2 mRNA were analyzed by normalizing with GAPDH mRNA expression.

\section{Western blotting}

Treated cells were washed twice with cold Tris-buffered saline, harvested using a cell scraper and lysed in buffer containing Tris (20 mM, pH 7.0), sodium $\beta$-glycerophosphate (25 mM), ethylene glycol tetraacetic acid (2 mM), Triton X-100 (1\%), phenylmethylsulfonyl fluoride (1 mM), aprotinin (1\%), dithiothreitol (2 mM) and vanadate (1 mM) on ice. Lysates were centrifuged at $17,000 \mathrm{~g}$ for $30 \mathrm{~min}$ at $4^{\circ} \mathrm{C}$. After normalization of protein concentrations, supernatants were mixed in equal amounts with a sample loading buffer. After denaturation by 
boiling at $100^{\circ} \mathrm{C}$ for $5 \mathrm{~min}$, samples were loaded onto a SDS-polyacrylamide gel, separated electrophoretically, and transferred to a polyvinylidene fluoride membrane (Millipore, Bedford, MA, USA). The membranes were incubated for $1 \mathrm{~h}$ with Tris-buffered saline containing $0.1 \%$ Tween 20 and 5\% dehydrated skim milk to block nonspecific binding. Subsequently, the membranes were probed with primary antibody [anti-phospho-p38 MAPK (\#4631, 1:1,000 dilution, Cell Signaling Technology, Danvers, MA, USA), anti-p38 MAPK (\#9212, 1:1,000 dilution, Cell Signaling Technology), anti-phospho-Akt (\#9271, 1:1,000 dilution, Cell Signaling Technology), anti-Akt (\#9272, 1:1,000 dilution, Cell Signaling Technology), anti- $\gamma$-GCS (\#RB-1697, 1:1,000 dilution, Neomarkers, Fremont, CA), HO-1 (\#SPA-895, 1:100,000 dilution, Stressgen, Victoria, Canada), anti- $\beta$-actin (\#A1978, 1:20,000 dilution, Sigma)] and with horseradish peroxidase-conjugated secondary antibody (1:1,000 dilution, GE Healthcare, Waukesha, WI, USA) for $1 \mathrm{~h}$. The membrane-bound secondary antibody was detected with an enhanced chemiluminescence detection system (ECL, GE Healthcare, Buckinghamshire, UK). The band intensities were analyzed with computer software, ImageJ 1.33u (National Institute for Health, Bethesda, MD, USA).

\section{Measurement of intracellular reduced glutathione (GSH) levels}

The levels of GSH were determined using monochlorobimane (MCB, Sigma), which forms a fluorescent conjugate together with GSH. PC12 cells were incubated with Krebs-Ringer-HEPES buffer (125 mM NaCl, 4.8 mM KCl, 25 mM HEPES, $1.2 \mathrm{mM} \mathrm{MgSO}_{4}$, $1.2 \mathrm{mM} \mathrm{KH}_{2} \mathrm{PO}_{4}, 5.6 \mathrm{mM}$ glucose, $2.2 \mathrm{mM} \mathrm{CaCl}_{2}$, pH7.4) containing MCB $(50 \mu \mathrm{M})$ for 30 min, and lysed in Triton X-100 (1\%). The fluorescence intensity of the lysates was measured 
using a spectrofluorometer (Ex 355 nm, Em 460 nm).

\section{Detection of intracellular ROS}

Levels of intracellular ROS were measured by flow cytometry and microscopic analysis as the fluorescence of 2',7'-dichlorofluorescein (DCF) and ethidium (ETH), which are the oxidation products of 2',7'-dichlorodihydrofluorescein $\left(\mathrm{H}_{2} \mathrm{DCF}\right)$ and dihydroethidium (DHE). $\mathrm{H}_{2} \mathrm{DCF}$ is more sensitive to hydrogen peroxide and hydroxyl radicals than to superoxide anions, whereas DHE is particularly sensitive to superoxide anions. $\mathrm{H}_{2}$ DCFDA (Molecular Probes, Eugene, OR, USA) is a diacetylated form of $\mathrm{H}_{2} \mathrm{DCF}$ that is freely membrane permeable and enters the cells. After entering the cells, the diacetyl groups of $\mathrm{H}_{2}$ DCFDA are cleaved by intracellular esterases and the resulting $\mathrm{H}_{2}$ DCF can be oxidized to highly fluorescent DCF by ROS. DHE (Sigma) is a lipophilic cell-permeable dye that can undergo oxidation to red fluorescent ETH in the presence of ROS. ETH then binds irreversibly to the double-stranded DNA causing amplification of the red fluorescent signal.

For flow cytometry, treated cells were incubated for 30 min with the probe ( $\mathrm{H}_{2} \mathrm{DCFDA}: 30 \mu \mathrm{M}$, and DHE: $10 \mu \mathrm{M}$ ) at $37^{\circ} \mathrm{C}$. A FACScan (Becton-Dickinson, Rutherford, NJ, USA) flow cytometer, equipped with a 488-nm argon ion laser and supplied with the Cell Quest software, was applied to measure ROS levels in the cells. Signals were obtained using a 530-nm bandpass filter (FL-1 channel) for DCF and a 585-nm bandpass filter (FL-2 channel) for ETH. Each determination was based on the mean fluorescence intensity of 1,000 cells. For microscopic analysis, cells were incubated with $\mathrm{H}_{2}$ DCFDA $(10 \mu \mathrm{M})$ and a cell-permeable nuclear indicator Hoechst 33342 (100 $\mu \mathrm{g} / \mathrm{mL}$; Molecular Probes) for $30 \mathrm{~min}$ at $37^{\circ} \mathrm{C}$. The cells 
were rinsed twice with phenol red-free DMEM, and then treated with 6-OHDA. Fluorescence was visualized using an Olympus IX81 inverted microscope and Metamorph software.

For flow cytometry, $\mathrm{H}_{2} \mathrm{DCFDA}$ was used at $30 \mu \mathrm{M}$, a concentration that permitted the quantification of baseline fluorescence as well as peak fluorescence without saturation. For microscopic analysis, however, the images at peak fluorescence were saturated because of a limited dynamic range. It was therefore necessary to reduce the concentration of $\mathrm{H}_{2}$ DCFDA to $10 \mu \mathrm{M}$. At this concentration, peak images were not saturated, but baseline levels were too low to be quantified with precision.

\section{Statistics}

The statistical significance of the differences between three or more groups was analyzed with a one-way analysis of variance (ANOVA) and post hoc multiple comparison using Turkey's test, unless otherwise stated. Statistical significance was defined as $p<0.05$. Data are expressed as the mean \pm standard error of the mean (SEM). 


\section{Results}

\section{Comparison of ARE activity between the extracts of fruits and vegetables}

To evaluate the effects of fruit and vegetable extracts on the cellular defense response against oxidative stress, we established rat PC12 stable reporter cell lines expressing the luciferase coding sequences controlled by ARE from the rat NQO 1 gene. At first, PC12 reporter cells were treated with the ether extracts of fruits and vegetables for $9 \mathrm{~h}$. The ether extracts of cranberry (juice) and green perilla (raw leaves) significantly increased luciferase activity (Fig. 1A), although cell death was not apparent at this time point. At the same concentration, native PC12 cells were treated with the ether extracts of fruits and vegetables for $48 \mathrm{~h}$. The ether extracts of cranberry (juice), green perilla (raw leaves) and tossa jute (raw leaves) significantly decreased cell viability (Fig. 1B). Because the ether extract of cranberry, in particular, exhibited a potent cytotoxic effect, further luciferase assay-guided chromatographic separation was carried out on the ether extract of green perilla. The ether extracts of green perilla increased luciferase activity in a concentration-dependent manner (Fig. 1C). An ethanol extract (25-100\%) of green perilla (raw leaves) was evaluated for ARE-dependent transcriptional activity. The higher the ethanol concentration was, the higher the luciferase activity of the ethanol extract (Fig. 1D).

\section{Bioactivity-guided purification of the ARE activator from green perilla}

The ether extract of green perilla (raw leaves) was subjected to a silica gel column chromatography using $n$-hexane-ethyl acetate $(25 \% \rightarrow 50 \% \rightarrow 75 \% \rightarrow 100 \%$ ethyl acetate) to separate 12 fractions; among them, fraction 8 exhibited significantly increased luciferase activity (Fig. 2A). The ether extract of green perilla (raw leaves) was also subjected to reversed-phase HPLC with a C18 column and a water/acetonitrile solvent system (mobile-phase A, 99:1; 
mobile-phase B, 1:99) containing $0.1 \%$ TFA under a gradient of $1-100 \%$ mobile-phase B for 30 min (Fig. 2B). Fraction 18 exhibited significantly increased luciferase activity (Fig. 2C). The eluted active fraction 18 was collected and subjected to the second purification step. In the second purification by HPLC under an isocratic condition of 30\% of mobile-phase B, significantly increased luciferase activities were observed in fractions 34-36 (Fig. 2D and E). An aqueous extract after ether/water extraction of green perilla was also subjected to reversed-phase HPLC under a gradient of 1-100\% mobile-phase B for 30 min, but there were no active fractions (data not shown).

\section{Structure elucidation of the ARE activator from green perilla}

The active fractions 34-36 at the second purification step were collected and subjected to the third preparative HPLC with a water/acetonitrile solvent system (mobile-phase A, water; mobile-phase B, acetonitrile) under a gradient of $40-45 \%$ of mobile-phase B for $10 \mathrm{~min}$. The main peak collected showed a single band in thin layer chromatography (TLC) analysis (data not shown). By this method, $11 \mathrm{mg}$ of this compound was obtained from $100 \mathrm{~g}$ fresh weight of green perilla leaves. Furthermore, high-resolution MS analysis demonstrated that the molecular formula of this compound was $\mathrm{C}_{17} \mathrm{H}_{16} \mathrm{O}_{5}$ (observed: 300.0985, calculated mass: 300.0988). NMR studies $\left({ }^{1} \mathrm{H},{ }^{13} \mathrm{C}\right.$, DEPT, TOCSY, HMQC, and HMBC) revealed that the chemical structure of this compound was 2',3'-dihydroxy-4',6'-dimethoxychalcone (DDC; Fig. 3A), previously reported by Ichino et al. [25]. DDC has been previously isolated from leaves of Uvaria dulcis and whole plants of Sarcandra hainanensis, but pharmacological properties of the compound have not yet been investigated [26] and [27]. 


\section{Activation of ARE-dependent transcription by DDC}

DDC was synthesized from 2'-hydroxy-4',6'-dimethoxyacetophenone and trans-cinnamoyl chloride by Friedel-Crafts reaction [25]. ${ }^{1} \mathrm{H}-\mathrm{NMR}$ analysis demonstrated that synthetic DDC showed identical chemical shifts and peak pattern with the isolated compound (data not shown). Synthetic DDC was tested for its effect on ARE activity. Treatment of PC12 reporter cells with DDC resulted in a significant increase in luciferase activity. The effect of DDC on ARE activity was concentration-dependent and peaked at 9-12 h (Fig. 4A and B). At concentrations ( $<30 \mu \mathrm{M})$ that had enough ARE activity, exposure to DDC for $48 \mathrm{~h}$ exhibited no cytotoxic effect (Fig. 4C). A structure-activity relationship of chalcone derivatives (Fig. 3B and C) was investigated to clarify the structural requirements for DDC. Chalcone (compound $\mathbf{1}$ ) without any substituted group and cinnamaldehyde (compound 8), a substructure of chalcone, exhibited a slight increase in luciferase activity. Among the tested chalcone derivatives, dimethoxychalcone, including DDC, compound 6, and compound 7 had comparatively potent activity (Fig. 4D). Sulforaphane (3 $\mu \mathrm{M})$, a well-known ARE activator, was used as a positive control. Treatment of PC12 cells with sulforaphane (10 $\mu \mathrm{M}$ and more) for $48 \mathrm{~h}$ exhibited cytotoxicity (data not shown). Thus, we successfully identified DDC as the active compound responsible for the induction of ARE-dependent transcription in green perilla.

\section{Regulation of DDC-induced ARE-dependent transcriptional activation}

In general, binding of Nrf2 to ARE is necessary for antioxidant gene expression. To confirm the involvement of Nrf2 in DDC-induced ARE activation, PC12 cells were transfected 
with siRNAs against Nrf2. Quantitative real-time PCR analysis demonstrated that the mRNA level of Nrf2 was partially decreased by Nrf2 siRNA (Fig. 5A). The increase in luciferase activity by DDC was also partially suppressed by transfection of siRNA directed against Nrf2 (Fig. 5B). It has been reported that the phosphorylation of Nrf2 plays an important role in nuclear translocation and transcriptional activation through ARE. Our previous report showed that nuclear translocation of Nrf2 in PC12 cells was mediated via phosphorylation by protein kinases [28]. After treatment with DDC, phosphorylated p38 mitogen-activated protein kinase (MAPK) and phosphorylated Akt were transiently increased around the 1-h time point (Fig. 5C and D). Furthermore, the increase in luciferase activity by DDC was suppressed by SB203580, an inhibitor of p38 MAPK, and LY294002, an inhibitor of phosphoinositide 3-kinase (PI3K), in an additive manner (Fig. 5E).

\section{Induction of antioxidant enzymes by DDC}

The Nrf2-ARE pathway is critical in mediating the induction of $\gamma$-GCS, a rate-limiting enzyme in glutathione synthesis, NQO1, and HO-1. The induction of these antioxidant enzymes by DDC in PC12 cells was investigated. Treatment with DDC for $24 \mathrm{~h}$ increased $\gamma$-GCS protein level, GSH content, NQO1 activity and HO-1 protein level at concentrations that are comparable with those that increased ARE-dependent luciferase activity (Fig. 6A, C, E, and G). The effects of DDC on $\gamma$-GCS protein level, GSH content, and NQO1 activity were suppressed by co-treatment with SB203580 (Fig. 6B, D, and F). The effect of DDC on HO-1 protein level was not suppressed by either SB203580 or LY294002 (Fig. 6H and I), but decreased by a combination of SB203580 and LY294002 (Fig. 6J). 


\section{Cytoprotective effect of DDC against 6-OHDA-induced cytotoxicity}

6-OHDA is extensively used to induce cell death through oxidative processes in different cell types, including human neuroblastoma SH-SY5Y cells, PC12 cells and rat ventral mesencephalic dopaminergic neurons [28], [29], and [30]. To test the protective effect of DDC on oxidative stress, PC12 cells were challenged with 6-OHDA. Pre-treatment with DDC for 24 $\mathrm{h}$ alone provided protection against 6-OHDA-induced cytotoxicity to a similar extent as pre-treatment followed by co-treatment during the toxin exposure. However, co-treatment with DDC slightly exacerbated 6-OHDA-induced cytotoxicity (Fig. 7A). Pre-treatment with DDC provided protection at concentrations that are comparable with those that increased ARE-dependent luciferase activity (Fig. 7B). The cytoprotective effect of DDC was suppressed by SB203580 (Fig. 7C). To confirm the indirect antioxidant properties of DDC, intracellular ROS levels were measured using oxidant-sensitive dyes ( $\mathrm{H}_{2} \mathrm{DCFDA}$ and DHE). Pre-treatment with DDC for $24 \mathrm{~h}$ suppressed the increase in intracellular ROS levels induced by 6-OHDA (Fig. 7D-F). In particular, DDC decreased the basal level of DCF-sensitive ROS (Fig. 7E). Therefore, our results suggest that the cytoprotection of DDC is mediated by indirect antioxidant properties via ARE activation. 


\section{Discussion}

In this study, we compared the effects of various fruit and vegetable ether extracts on ARE-dependent transcriptional activation. Green perilla extract exhibited a high level of ARE activity. Furthermore, we identified DDC from the extract as an active ingredient responsible for the ARE activity. Our data demonstrated that DDC enhanced the induction of antioxidant enzymes through activation of the Nrf2-ARE pathway and provided protective action against 6-OHDA-induced cytotoxicity.

Perilla (Perilla frutescens; Labiatae) is an annual herbaceous plant native to Asia. There are two forms, the green form (green perilla) and the red form (red perilla), which differ in their accumulation of anthocyanins [31]. It has been reported that perilla leaves and seeds contained the anti-allergic, anti-inflammatory, anti-carcinogenic, and anti-human immunodeficiency virus substances, such as rosmarinic acid and $\alpha$-linolenic acid [32], [33], [34], and [35]. Because of their high abundance of antioxidant polyphenolic compounds, perilla leaf water extracts possessed potent radical scavenging activity [36]. In addition, we show that green perilla leaf ether extract potentiates the cellular defense system against oxidative stress. Although we showed that red perilla juice failed to increase ARE activity, red perilla leaf ether extract possessed ARE activation potency, although the effect was not so potent as green perilla leaf extract (data not shown). Considering that ARE activity of green perilla ethanol extracts increased in proportion to ethanol concentration and that green perilla water extract failed to increase ARE activity, it is suggested that the active ingredients responsible for ARE activation in perilla have comparatively low solubility in water.

Chalcone is a class of flavonoid compounds that are widely biosynthesized in plants 
and exhibits a basic structure of two benzene rings linked through an $\alpha$, $\beta$-unsaturated carbonyl group. Chalcone derivatives possess a diverse spectrum of biological activities, including anti-oxidative, anti-inflammatory, anti-cancer, and immunosuppressive potential [37], [38], and [39]. DDC has been previously isolated and identified from leaves of Uvaria dulcis and whole plants of Sarcandra hainanensis [26] and [27]. However, to our knowledge, there are no reports on the existence of DDC in green perilla, which is a dietary vegetable. Furthermore, the present study is the first report on the biological activity of DDC.

The present study provides the first evidence that DDC intensively induces Nrf2-dependent ARE activation. The activation of the Nrf2-ARE pathway by various electrophiles and compounds that are acceptors for Michael reactions is attributed to changes in redox environment and/or direct cysteine modification in Keap1 [40]. The $\alpha, \beta$-unsaturated carbonyl moiety in the chalcone skeleton provides the basis for the reaction of a Michael acceptor with a nucleophile. Previous study found that the $\alpha, \beta$-unsaturated carbonyl moiety was crucial for nucleophilic addition reactions with thiols and for increasing intracellular ROS levels [41]. Talalay et al. [42] demonstrated that many $\alpha, \beta$-unsaturated carbonyl compounds induced antioxidant enzymes and that the potency of inducers paralleled their efficiency in Michael reactions. Although the $\alpha, \beta$-unsaturated carbonyl moiety in chalcone plays a basic role in ARE activation, the present structure-activity relationship study revealed that the substituted groups on chalcone were of critical importance for potency. In addition, depending on cell type and inducer, multiple signaling kinases have been reported to regulate the Nrf2-ARE pathway, which include p38 MAPK, extracellular signal-regulated kinase, c-jun $\mathrm{NH}_{2}$-terminal kinase, PI3K and protein kinase C [43]. Our findings indicate that p38 MAPK pathway and PI3K/Akt 
pathway additively participated in ARE activation induced by DDC. Further studies are needed to elucidate the mechanisms of Nrf2-dependent ARE activation induced by DDC.

Our data demonstrated that DDC provided protection against 6-OHDA-induced cytotoxicity. The cytoprotection of DDC against 6-OHDA toxicity did not require co-treatment but pre-treatment before the toxin challenge. The mechanisms underlying the effectiveness of DDC against 6-OHDA cytotoxicity are not completely known, but the indirect antioxidative properties of DDC could explain its protective effect. We showed that DDC was able to completely reverse the drastic increase in ROS formation induced by 6-OHDA. DDC increased antioxidative enzymes, such as $\gamma$-GCS, NQO1, and HO-1 in a concentration-dependent manner. The cytoprotection induced by DDC in accordance with the induction of $\gamma$-GCS, GSH, and NQO1 was abolished by inhibition of p38 MAPK pathway. These results suggest that the cytoprotection of DDC may be mediated by the antioxidant enzymes up-regulated by p38 MAPK-dependent ARE activation. 6-OHDA is rapidly oxidized by molecular oxygen to form superoxide anions, hydrogen peroxide, and the corresponding $p$-quinone, which contribute to the cytotoxicity [27]. GSH scavenge ROS and interact with 6-OHDA-oxidized products, forming glutathionyl conjugates [44]. NQO1 catalyzes the two-electron reduction of quinones to hydroquinones, which prevents the formation of highly reactive semiquinones. In addition, catalase and superoxide dismutase have been reported to be up-regulated by ARE activation [45]. Therefore, antioxidant enzymes regulated via the Nrf2-ARE pathway may comprehensively participate in the cytoprotection of DDC. Elevation of HO-1 levels by DDC was not abolished by inhibition of either p38 MAPK pathway or PI3K/Akt pathway. However, inhibition of both p38 MAPK pathway and PI3K/Akt pathway suppressed the induction of 
HO-1 levels. Therefore, these pathways may serve as a compensatory mechanism for HO-1 upregulation. In addition, since the treatment with DDC (30 $\mu \mathrm{M})$ in the presence of both inhibitors of p38 MAPK and PI3K for $24 \mathrm{~h}$ was injurious to cells (data not shown), elevation of HO-1 levels by DDC seems to play an important role in defensive mechanisms against DDC-induced cell damage.

In summary, we succeeded in isolating and identifying the Nrf2-ARE activator from green perilla ether extract. DDC was found to induce the expression of various antioxidant proteins, suppress intracellular ROS formation, and thereby enhance cellular resistance to 6-OHDA-induced cytotoxicity. Previous studies have described the nuclear localization of Nrf2 in patients with Parkinson disease and Nrf2-mediated neuroprotection in models of Parkinson disease [46], [47], and [48]. Therefore, DDC may have the potential to be developed as a therapeutic agent aimed at reducing or preventing cell death in Parkinson disease. Therefore, we propose that indirect as well as direct antioxidant activity of dietary fruits and vegetables are beneficial to human health. 
Acknowledgements: This work was supported by grants-in-aid for Scientific Research from the Japan Society for the Promotion of Science and from the Ministry of Education, Culture, Sports, Science and Technology of Japan. We thank our colleagues for helpful discussions. 


\section{References}

[1] Diaz MN, Frei B, Vita JA, Keaney JF Jr. Antioxidants and atherosclerotic heart disease. N Engl J Med. 1997 337:408-416.

[2] Giasson BI, Ischiropoulos H, Lee VM, Trojanowski JQ. The relationship between oxidative/nitrative stress and pathological inclusions in Alzheimer's and Parkinson's diseases. Free Radic Biol Med. 2002 32:1264-1275.

[3] Klaunig JE, Kamendulis LM. The role of oxidative stress in carcinogenesis. Annu Rev Pharmacol Toxicol. 2004;44:239-67.

[4] Olinski R, Siomek A, Rozalski R, Gackowski D, Foksinski M, Guz J, Dziaman T, Szpila A, Tudek B. Oxidative damage to DNA and antioxidant status in aging and age-related diseases. Acta Biochim Pol. 2007 54:11-26.

[5] Massy ZA, Stenvinkel P, Drueke TB. The role of oxidative stress in chronic kidney disease. Semin Dial. 2009 22:405-408.

[6] Roberts C.K., Sindhu K.K., Oxidative stress and metabolic syndrome, Life Sci. 2009 84:705-712.

[7] Joshipura KJ, Ascherio A, Manson JE, Stampfer MJ, Rimm EB, Speizer FE, Hennekens CH, Spiegelman D, Willett WC. Fruit and vegetable intake in relation to risk of ischemic stroke. JAMA. 1999 282:1233-1239.

[8] Riboli E, Norat T. Epidemiologic evidence of the protective effect of fruit and vegetables on cancer risk. Am J Clin Nutr. 2003 78:559S-569S.

[9] Dai Q, Borenstein AR, Wu Y, Jackson JC, Larson EB. Fruit and vegetable juices and Alzheimer's disease: the Kame Project. Am J Med. 2006 119:751-759. 
[10] Gale CR, Martyn CN, Winter PD, Cooper C. Vitamin C and risk of death from stroke and coronary heart disease in cohort of elderly people. BMJ. 1995 310:1563-1566.

[11] Morris MC, Beckett LA, Scherr PA, Hebert LE, Bennett DA, Field TS, Evans DA. Vitamin E and vitamin C supplement use and risk of incident Alzheimer disease. Alzheimer Dis Assoc Disord. 1998 12:121-126.

[12] Le Marchand L, Murphy SP, Hankin JH, Wilkens LR, Kolonel LN. Intake of flavonoids and lung cancer. J Natl Cancer Inst. 2000 92:154-160.

[13] Vokó Z, Hollander M, Hofman A, Koudstaal PJ, Breteler MM. Dietary antioxidants and the risk of ischemic stroke: the Rotterdam Study. Neurology. 2003 61:1273-1275.

[14] Cao, G; Alessio, H.; Cutler, R. Oxygen-radical absorbence capacity assay for antioxidants. Free Radical Biol. Med. 1993, 14, 303-311.

[15] Miller NJ, Rice-Evans C, Davies MJ, Gopinathan V, Milner A. A novel method for measuring antioxidant capacity and its application to monitoring the antioxidant status in premature neonates. Clin Sci (Lond). 1993 84:407-412.

[16] Benzie, I. F. F.; Strain, J. J. The ferric reducing ability of plasma (FRAP) as a measure of “antioxidant power”: the FRAP assay. Anal. Biochem. 1996, 239, 70-76.

[17] Halliwell B, Rafter J, Jenner A. Health promotion by flavonoids, tocopherols, tocotrienols, and other phenols: direct or indirect effects? Antioxidant or not? Am J Clin Nutr. 2005 81:268S-276S.

[18] Dinkova-Kostova AT, Talalay P. Direct and indirect antioxidant properties of inducers of cytoprotective proteins. Mol Nutr Food Res. 2008 52:S128-138. 
[19] Motohashi H, Yamamoto M. Nrf2-Keap1 defines a physiologically important stress response mechanism. Trends Mol Med. 2004 10:549-557.

[20] Jeong WS, Jun M, Kong AN. Nrf2: a potential molecular target for cancer chemoprevention by natural compounds. Antioxid Redox Signal. 2006 8:99-106.

[21] Li J, Ichikawa T, Janicki JS, Cui T. Targeting the Nrf2 pathway against cardiovascular disease. Expert Opin Ther Targets. 2009 13:785-794.

[22] Scapagnini G, Vasto S, Abraham NG, Caruso C, Zella D, Fabio G Modulation of Nrf2/ARE pathway by food polyphenols: a nutritional neuroprotective strategy for cognitive and neurodegenerative disorders. Mol Neurobiol. 2011 44:192-201.

[23] Prochaska HJ, Santamaria AB, Talalay P. Rapid detection of inducers of enzymes that protect against carcinogens. Proc Natl Acad Sci U S A. 1992 892394-1298.

[24] Kume T, Asai N, Nishikawa H, Mano N, Terauchi T, Taguchi R, Shirakawa H, Osakada F, Mori H, Asakawa N, Yonaga M, Nishizawa Y, Sugimoto H, Shimohama S, Katsuki H, Kaneko S, Akaike A. Isolation of a diterpenoid substance with potent neuroprotective activity from fetal calf serum. Proc Natl Acad Sci U S A. 2002 99:3288-3293.

[25] Ichino K, Tanaka H, Ito K, Tanaka T, Mizuno M. Synthesis of helilandin B, pashanone, and their isomers. J Nat Prod. 1988 51:906-914.

[26] Chantrapromma K, Rat-A-pa Y, Karalai C, Lojanapiwatana V, Seechamnanturakit V. A chalcone and a dihydrochalcone from Uvaria dulcis. Phytochemistry. 2000 53:511-513.

[27] Cao C, Xu L, Chen K, Peng Y, Xiao P. Chemical study on petroleum ether portion of Sarcandra hainanensis. Zhongguo Zhong Yao Za Zhi. 2009 34:1009-1010. 
[28] Yamamoto N, Sawada H, Izumi Y, Kume T, Katsuki H, Shimohama S, Akaike A.

Proteasome inhibition induces glutathione synthesis and protects cells from oxidative stress: relevance to Parkinson disease. J Biol Chem. 2007 282:4364-4372.

[29] Izumi Y, Sawada H, Sakka N, Yamamoto N, Kume T, Katsuki H, Shimohama S, Akaike A. p-Quinone mediates 6-hydroxydopamine-induced dopaminergic neuronal death and ferrous iron accelerates the conversion of p-quinone into melanin extracellularly. J Neurosci Res. 2005 79:849-860.

[30] Yamamoto N, Izumi Y, Matsuo T, Wakita S, Kume T, Takada-Takatori Y, Sawada H, Akaike A. Elevation of heme oxygenase-1 by proteasome inhibition affords dopaminergic neuroprotection. J Neurosci Res. 2010 88:1934-1942.

[31] Saito K, Yamazaki M. Biochemistry and molecular biology of the late-stage of biosynthesis of anthocyanin: lessons from Perilla frutescens as a model plant. New Phytol 2002 155:9-23.

[32] Kawahata T, Otake T, Mori H, Kojima Y, Oishi I, Oka S, Fukumori Y, Sano K. A novel substance purified from Perilla frutescens Britton inhibits an early stage of HIV-1 replication without blocking viral adsorption. Antivir Chem Chemother. 2002 13:283-288.

[33] Osakabe N, Yasuda A, Natsume M, Yoshikawa T. Rosmarinic acid inhibits epidermal inflammatory responses: anticarcinogenic effect of Perilla frutescens extract in the murine two-stage skin model. Carcinogenesis. 2004 25:549-575.

[34] Chang HH, Chen CS, Lin JY. Dietary perilla oil inhibits proinflammatory cytokine production in the bronchoalveolar lavage fluid of ovalbumin-challenged mice. Lipids. 2008 
43:499-506.

[35] Oh HA, Park CS, Ahn HJ, Park YS, Kim HM. Effect of Perilla frutescens var. acuta Kudo and rosmarinic acid on allergic inflammatory reactions. Exp Biol Med (Maywood). 2011 236:99-106.

[36] Meng L, Lozano YF, Gaydou EM, Li B. Antioxidant activities of polyphenols extracted from Perilla frutescens varieties. Molecules. 2008 14:133-140.

[37] Kumar V, Kumar S, Hassan M, Wu H, Thimmulappa RK, Kumar A, Sharma SK, Parmar VS, Biswal S, Malhotra SV. Novel chalcone derivatives as potent Nrf2 activators in mice and human lung epithelial cells. J Med Chem. 2011 Jun 23;54(12):4147-59. Epub 2011 May 19.

[38] Wu J, Li J, Cai Y, Pan Y, Ye F, Zhang Y, Zhao Y, Yang S, Li X, Liang G Evaluation and discovery of novel synthetic chalcone derivatives as anti-inflammatory agents. J Med Chem. 2011 54:8110-8123.

[39] Yadav VR, Prasad S, Sung B, Aggarwal BB. The role of chalcones in suppression of NF-кB-mediated inflammation and cancer. Int Immunopharmacol. 2011 11:295-309.

[40] Holtzclaw WD, Dinkova-Kostova AT, Talalay P. Protection against electrophile and oxidative stress by induction of phase 2 genes: the quest for the elusive sensor that responds to inducers. Adv Enzyme Regul. 2004;44:335-67.

[41] Shibata T, Yamada T, Ishii T, Kumazawa S, Nakamura H, Masutani H, Yodoi J, Uchida K. Thioredoxin as a molecular target of cyclopentenone prostaglandins. J Biol Chem. 2003 $278: 26046-26054$.

[42] Talalay P, De Long MJ, Prochaska HJ. Identification of a common chemical signal 
regulating the induction of enzymes that protect against chemical carcinogenesis. Proc Natl Acad Sci U S A. 1988 85:8261-8265.

[43] Keum YS, Jeong WS, Kong AN. Chemoprevention by isothiocyanates and their underlying molecular signaling mechanisms. Mutat Res. 2004 555:191-202.

[44] Nappi AJ, Vass E. 1994. The effects of glutathione and ascorbic acid on the oxidations of 6-hydroxydopamine. Biochim Biophys Acta 1201:498-504.

[45] Zhu H, Itoh K, Yamamoto M, Zweier JL, Li Y. Role of Nrf2 signaling in regulation of antioxidants and phase 2 enzymes in cardiac fibroblasts: protection against reactive oxygen and nitrogen species-induced cell injury. FEBS Lett. 2005 579:3029-3036.

[46] Ramsey CP, Glass CA, Montgomery MB, Lindl KA, Ritson GP, Chia LA, Hamilton RL, Chu CT, Jordan-Sciutto KL. Expression of Nrf2 in neurodegenerative diseases. J Neuropathol Exp Neurol. 2007 66:75-85.

[47] Jakel RJ, Townsend JA, Kraft AD, Johnson JA. Nrf2-mediated protection against 6-hydroxydopamine. Brain Res. 2007 1144:192-201.

[48] Chen PC, Vargas MR, Pani AK, Smeyne RJ, Johnson DA, Kan YW, Johnson JA. Nrf2-mediated neuroprotection in the MPTP mouse model of Parkinson's disease: Critical role for the astrocyte. Proc Natl Acad Sci U S A. 2009 106:2933-2938. 


\section{Figure legends}

Fig.1 Effects of fruit and vegetable extracts on ARE activity. A: Effects of ether extracts of fruits and vegetables on ARE activity. PC12 reporter cells were treated with ether extracts for $9 \mathrm{~h}$. The concentrations of each extract were: peach (juice), $100 \mu \mathrm{L} / \mathrm{mL}$; apple (juice), $50 \mu \mathrm{L} / \mathrm{mL}$; strawberry (juice), $100 \mu \mathrm{L} / \mathrm{mL}$; cranberry (juice), $100 \mu \mathrm{L} / \mathrm{mL}$; raspberry (juice), $100 \mu \mathrm{L} / \mathrm{mL}$; satsuma mandarin (juice), $20 \mu \mathrm{L} / \mathrm{mL}$; green perilla (raw leaves), $50 \mathrm{mg} / \mathrm{mL}$; tossa jute (raw leaves), 25 mg/mL; crown daisy (raw leaves), 20 mg/mL; celery (juice), $100 \mu \mathrm{L} / \mathrm{mL}$; parsley (juice), $50 \mu \mathrm{L} / \mathrm{mL}$; red perilla (juice), $100 \mu \mathrm{L} / \mathrm{mL}$; crown daisy (juice), $100 \mu \mathrm{L} / \mathrm{mL}$. B: Effects of ether extracts of fruits and vegetables on cell viability. Native PC12 cells were treated with ether extracts for $48 \mathrm{~h}$. C: Concentration-dependent effect of ether extract of green perilla on ARE activity. PC12 reporter cells were treated with ether extracts of green perilla for $9 \mathrm{~h}$. D: Effects of ethanol extracts of green perilla on ARE activity. PC12 reporter cells were treated with ethanol extracts of green perilla for 9 h. ${ }^{\#} p<0.05,{ }^{\# \#} p<0.001$, compared with control.

Fig. 2 Chromatographic purification of active fraction from ether extract of green perilla. A: Effects of fractions separated by silica gel column chromatography on ARE activity. B: HPLC-UV (300 nm) chromatogram of the first purification step of green perilla ether extract. C: Effects of first HPLC fractions of green perilla ether extract on ARE activity. D: HPLC-UV (300 $\mathrm{nm}$ ) chromatogram of the second purification step of green perilla ether extract. E: Effects of second HPLC fractions of green perilla ether extract on ARE activity. PC12 reporter cells were treated with each fraction of green perilla for 9 h. ${ }^{\# \#} p<0.001$, compared with control. 
Fig. 3 Chemical structures of chalcone derivatives. A: 2',3'-Dihydroxy-4',6'-dimethoxychalcone (DDC). B: Chalcone derivatives. C: Cinnamaldehyde.

Fig. 4 Effect of synthetic DDC on ARE activity. A: Concentration-dependent effect of DDC on ARE activity. PC12 reporter cells were treated with DDC (3-30 $\mu \mathrm{M})$ for $9 \mathrm{~h}$. B:

Time-dependent effect of DDC on ARE activity. PC12 reporter cells were treated with DDC (30 $\mu \mathrm{M}$ ) for the indicated periods. Statistical analyses were performed using two-way ANOVA and post hoc multiple comparison using Bonferroni test. C: Effect of DDC on cell viability. Native PC12 cells were treated with DDC (1-100 $\mu \mathrm{M})$ for $48 \mathrm{~h}$. D: Effects of chalcone derivatives on ARE activity. PC12 reporter cells were treated with chalcone derivatives (30 $\mu \mathrm{M})$ or sulforaphane $(3 \mu \mathrm{M})$ for 9 h. ${ }^{\# \#} p<0.001$, compared with control.

Fig. 5 Mechanisms of ARE activation induced by DDC. A: Effect of Nrf2 siRNA on Nrf2 mRNA expression. Native PC12 cells were incubated for $48 \mathrm{~h}$ after transfection with siRNA against Nrf2. B: Effect of Nrf2 siRNA on ARE activation induced by DDC. PC12 reporter cells were incubated for $48 \mathrm{~h}$ after transfection, and then were treated with DDC (30 $\mu \mathrm{M})$ for a further 12 h. C,D: Effect of DDC on phosphorylation of p38 MAPK and Akt. Native PC12 cells were treated with DDC (30 $\mu \mathrm{M})$ for the indicated periods. E: Effects of p38 MAPK and PI3K inhibitors on ARE activation induced by DDC. PC12 reporter cells were treated with DDC (30 $\mu \mathrm{M})$ in the presence or absence of SB203580 and LY294002 (10-30 $\mu \mathrm{M})$ for 9 h. ${ }^{\# \#} p<0.001$ compared with control. ${ }^{* * *} p<0.001$ compared with DDC alone. 
Fig. 6 Effect of DDC on ARE-regulated antioxidant proteins. A, C, E, G: Effect of DDC on $\gamma$-GCS protein level (A), GSH content (C), NQO1 activity (E), and HO-1 protein level (G). Native PC12 cells were treated with DDC (3-30 $\mu \mathrm{M})$ for $24 \mathrm{~h}$. B, D, F: Effect of p38 MAPK inhibitor on DDC-induced elevation of $\gamma$-GCS protein level (B), GSH content (D), and NQO1 activity (F). Native PC12 cells were treated with DDC (30 $\mu \mathrm{M})$ in the presence or absence of SB203580 (10-30 $\mu \mathrm{M})$ for $24 \mathrm{~h}$. H-J: Effects of p38 MAPK and PI3K inhibitors on DDC-induced elevation of HO-1 protein level. Native PC12 cells were treated with DDC (30 $\mu \mathrm{M})$ in the presence or absence of SB203580 and LY294002 (10-30 $\mu \mathrm{M})$ for $24 \mathrm{~h}^{\text {\# }} \mathrm{p}<0.01$, ${ }^{\# \#} p<0.001$, compared with control. ${ }^{* * *} p<0.001$ compared with DDC alone.

Fig. 7 Effect of DDC on 6-OHDA-induced cytotoxicity. A: Time course study on the effect of DDC on 6-OHDA toxicity. Native PC12 cells were exposed to 6-OHDA (200 $\mu \mathrm{M})$ for $24 \mathrm{~h}$ with or without pre-treatment for $24 \mathrm{~h}$ and co-treatment with DDC (30 $\mu \mathrm{M})$. B:

Concentration-dependent effect of DDC on 6-OHDA toxicity. Native PC12 cells were treated with DDC (3-30 $\mu \mathrm{M})$ for $24 \mathrm{~h}$ prior to 6-OHDA exposure, and then exposed to 6-OHDA (200 $\mu \mathrm{M})$ for $24 \mathrm{~h}$. C: Effect of p38 MAPK inhibitor on DDC-induced cytoprotection against 6-OHDA toxicity. Native PC12 cells were treated with DDC (30 $\mu \mathrm{M})$ in the presence or absence of SB203580 (3-30 $\mu \mathrm{M}$ ) for $24 \mathrm{~h}$ prior to 6-OHDA exposure, and then were exposed to 6-OHDA $(200 \mu \mathrm{M})$ for $24 \mathrm{~h}$. D-F: Effect of DDC on 6-OHDA-induced ROS formation. D: Representative fluorescence microscope images from DCF fluorescence. Native PC12 cells were pretreated with DDC (30 $\mu \mathrm{M})$ for $24 \mathrm{~h}$, incubated with $\mathrm{H}_{2}$ DCFDA for $30 \mathrm{~min}$, and exposed to 6-OHDA (200 $\mu \mathrm{M})$ for $1 \mathrm{~h}$. Scale bar $=50 \mu \mathrm{m}$. E,F: Analysis of DCF and ETH 
fluorescence by flow cytometry. Native PC12 cells were treated with DDC (3-30 $\mu \mathrm{M})$ for $24 \mathrm{~h}$ prior to 6-OHDA exposure, and then were exposed to 6-OHDA $(200 \mu \mathrm{M})$ for 3 h. ${ }^{\#} p<0.05,{ }^{\# \#} p$ $<0.001$ compared with control. ${ }^{*} p<0.05, \stackrel{* *}{p}<0.01,{ }^{* * *} p<0.001$ compared with 6-OHDA alone. ${ }^{+1} p<0.001$ compared with 6-OHDA+DDC. 